\title{
Development of Electro-thermal Model for Quality Assurance of Materials
}

\author{
Merin Lizbeth George ${ }^{1 *}$, C. N. Ravi ${ }^{2}$, R. Harikrishnan ${ }^{2}$ and G. R. P. Lakshmi ${ }^{3}$ \\ 1PG Scholar, Sathyabama University, Chennai; merinz01@gmail.com \\ 2 Lecturer, Sathyabama University, Chennai \\ ${ }^{3}$ HOD of EEE Sathyabama University, Chennai
}

\begin{abstract}
This paper presents electro-thermal modeling using Resistance-Capacitance (RC) for transient heat conduction to inspect temperature variation over the surface of a material (AISI 316 steel) with defective and non-defective zones. An electrical based analytic approach for stepped infrared thermography is presented based on the correspondence between the fundamental laws of heat transfer and electricity. The construction of approximate analogous electrical models of thermal problem has been used in the mathematical analysis of heat transfer. A rigorous discussion of the heat dissipation mechanism over the material is performed, to show that the temperature over the defect region is always greater than the reference area and to prove the analogy of thermal to electrical parameter from the surface temperature evolution.
\end{abstract}

Keywords: Electro-thermal Modeling, Stepped Infrared Thermography, AISI 316 Steel, Analytic Approach, Analogy

\section{Introduction}

Active Infrared (IR) thermography is a non-destructive evaluation technique, employed to inspect the integrity of materials by the application of an external thermal stimulus. It is relatively fast, non intrusive, non contact and allows the detection of shallow subsurface defects [1]. The present work mainly based on stepped thermography, a form of active thermography, to provide uniform heating of the sample under test. In comparison with other thermographic techniques, stepped thermography can be employed with low intensity heat sources.

Analysis and simulation based on electrical modeling of heat transfer is extensively used for thermal investigation. Significant work has been done to study the propagation of thermal waves in solids and to analyze the thermal response of the material subjected to external heat stimuli [2]. Traditional approach in heat transfer converts the thermal problem into an electrical equivalent circuit for ease of understanding and to reduce the difficulties of purely analytical thermal approach.

Figure 1 depicts the generation of thermal image using active IR thermography setup and can be summarized as follows. The surface of the specimen is stimulated with radiative heat sources, which is controlled using a source control unit. When the heat flows inside the material it gets perturbed by the presence of hidden defects causing temperature contrast at the surface of the specimen. The temperature difference is recorded using an infrared camera synchronized with the computer and the thermogram is generated.

\section{Electro-thermal Approach}

The modeling approach is based on a simplified circuital representation to reduce the complexity of a problem in thermal field by analogous equivalent electrical model. The

\footnotetext{
* Corresponding author:

Merin Lizbeth George (merinz01@gmail.com)
} 


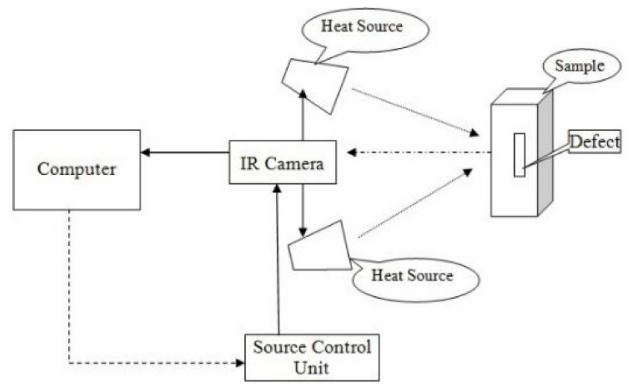

Figure1. Schematic of the data acquisition through active IR thermography setup.

thermal and electrical field can be described by similar equations and behavior as given in the table below.

Table 1. Thermal to electrical analogy

\begin{tabular}{lclc}
\hline $\begin{array}{l}\text { Thermal } \\
\text { quantities }\end{array}$ & Units & $\begin{array}{l}\text { Electrical } \\
\text { quantities }\end{array}$ & Units \\
\hline Heat $(\mathrm{q})$ & $\mathrm{W}$ & Current $(\mathrm{I})$ & $\mathrm{A}$ \\
Temperature & $\mathrm{K}$ & Voltage $(\mathrm{V})$ & $\mathrm{V}$ \\
$\begin{array}{l}\text { Thermal } \\
\text { Resistance }\left(\mathrm{R}_{\mathrm{th}}\right)\end{array}$ & $\mathrm{K} / \mathrm{W}$ & $\begin{array}{l}\text { Electrical } \\
\text { Resistance }(\mathrm{R})\end{array}$ & $\Omega$ \\
$\begin{array}{l}\text { Heat Stored } \\
\text { Absolute Zero } \\
\text { temperature }\end{array}$ & $\mathrm{J} / \mathrm{K}$ & Capacitor(C) & $\mathrm{F}$ \\
\hline
\end{tabular}

Based on the following correspondence [2]:

$$
\begin{aligned}
q & =\frac{\Delta T}{R_{t h}}(\text { heat transfer side }) \\
\leftrightarrow I & =\frac{\Delta V}{R} \text { (electrical side) }
\end{aligned}
$$

where $\mathrm{q}, \Delta T$ and $\mathrm{R}_{\mathrm{th}}$ are the rate of heat transfer, temperature difference and thermal resistance (in thermodynamic units) and I, $\Delta V$ and $R$ are the current, voltage difference and electrical resistance (in electrical units) respectively. Eq. (1) shows that the rate of heat transfer is analogous to electrical current.

A material of thickness $\Delta d$, cross-sectional area $A$, thermal conductivity $k$, density $\rho$ and specific heat $c$, can be electrically modeled by an equivalent resistance $R$ and capacitance $C$, using above equivalence between thermal and electrical parameter [4].

$$
\begin{aligned}
& R=\frac{d}{k A} \\
& C=\rho A d c
\end{aligned}
$$

An electrical analog of the thermal problem can be modeled as RC network for an easy judgment and for obtaining the solutions easily.

\section{Modeling and Analysis}

A three dimensional temperature prediction model had been made by Rajesh Gupta and Suneet Tuli [5]. When a defect is present in the material, it behaves as lumped over defect and non-defect regions and therefore, the information can be extracted easily from the lumped model. The transient heat conduction specimen is modeled by dividing it into small cuboids and the resistances and capacitances for different zones have been modeled using Eqs. (2) and (3).

Under a constant heat flux $F$, the transfer of heat through the material with thickness $d$, after the initial transient period represented as

$$
T=\frac{F t}{\rho c d}
$$

According to Eq. (4) the presence of a defect in the actual material causes rate of rise of temperature which is greater in the defect region due to its smaller thickness on comparing with the original zone. However this happens only initially, because material over the defect is connected laterally with non-defect material, due to which after sometime the temperature over the defect and non-defect regions increases linearly at the same rate. This saturates absolute thermal contrast [3].

In Figure 2, equivalent electro-thermal capacitances of the material, $C_{d}$ and $C_{n d}$ over the defect and non-defect regions respectively are connected between four equal

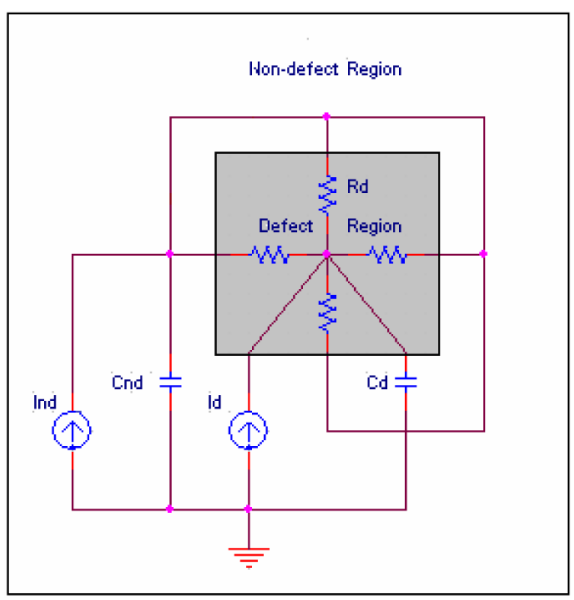

Figure 2. Lumped RC model (cross-section shown parallel to heated surface). 
parallel resistances $R_{d}$, corresponding to the thermal resistance over the defect region in lateral dimensions [5]. These capacitors are fed by step current sources $I_{d}$ and $I_{n d}$ of defect and non-defect zones. According to the Eqs. (1)-(3) a simplified electrical equivalent circuit for the above model is obtained with a single resistance $r$, equivalent to four parallel resistances $R_{d}$. For a square defect, the value of $r$ is independent of lateral dimensions of the defect.

Voltages at node $1\left(V_{d}\right)$ and node $2\left(V_{n d}\right)$ of Figure 3 are analogous to temperatures at the centre of the defect $T_{d}$ and the non-defect $T_{n d}$ regions, respectively. Applying Kirchhoff's law at nodes 1 and 2, and transforming to frequency domain results in

$$
\begin{gathered}
\frac{I_{d}}{s}=\frac{V_{d}-V_{n d}}{r}+V_{d} C_{d} s \\
\frac{I_{n d}}{s}+\frac{V_{d}-V_{n d}}{s}=V_{n d} C_{n d} s
\end{gathered}
$$

$V_{d}$ and $V_{n d}$, in the frequency domain can be obtained by using the above equations,

$$
\begin{gathered}
V_{d}(s)=\frac{I_{d}+I_{n d}+I_{d} C_{n d} s r}{s^{2}\left(C_{d}+C_{n d}+C_{d} C_{n d} s r\right)} \\
V_{n d}(s)=\frac{I_{d}+I_{n d}+I_{n d} C_{n d} s r}{s^{2}\left(C_{d}+C_{n d}+C_{d} C_{n d} s r\right)}
\end{gathered}
$$

The above equations can be converted into time domain by taking the inverse Laplace transform, provides $V_{d}$ and $V_{n d}$,

$$
\begin{aligned}
V_{d}(t) & =\frac{\left(I_{d}+I_{n d}\right) t}{\left(C_{d}+C_{n d}\right)} \\
& +\frac{r C_{n d}\left(C_{n d} I_{d}-C_{d} I_{n d}\right)\left(1-\exp \left(-\frac{\left(C_{d}+C_{n d}\right) t}{r C_{d} C_{n d}}\right)\right)}{\left(C_{d}+C_{n d}\right)^{2}}
\end{aligned}
$$

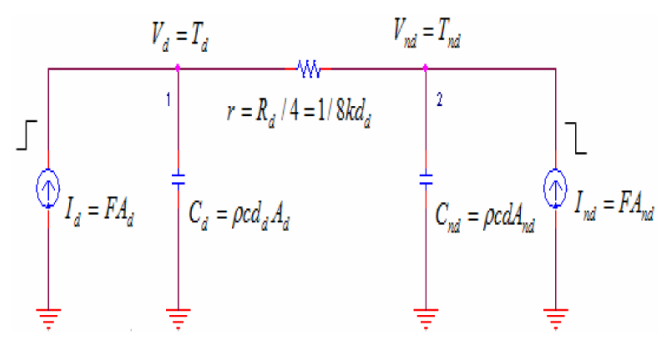

Figure 3. Simplified electrical model with equivalent parameters.

$$
\begin{aligned}
V_{n d}(t) & =\frac{\left(I_{d}+I_{n d}\right) t}{\left(C_{d}+C_{n d}\right)} \\
& +\frac{r C_{d}\left(C_{n d} I_{d}-C_{d} I_{n d}\right)\left(1-\exp \left(-\frac{\left(C_{d}+C_{n d}\right) t}{r C_{d} C_{n d}}\right)\right)}{\left(C_{d}+C_{n d}\right)^{2}}
\end{aligned}
$$

Each of these equations has two terms: one varying term and other exponentially with time, similar to the equation for charging a capacitor through a resistance. The difference between Eqs. (9) and (10) will correspond to the absolute thermal contrast, which is given by

$$
\begin{aligned}
& V_{d}(t)-V_{n d}(t)= \\
& \frac{r\left(C_{n d} I_{d}-C_{d} I_{n d}\right)\left(1-\exp \left(-\frac{\left(C_{d}+C_{n d}\right) t}{r C_{d} C_{n d}}\right)\right)}{C_{d}+C_{n d}}
\end{aligned}
$$

Generally, the defect area is much smaller than non-defect area. Using this condition $(A d<<A n d)$ with thermal equivalence of quantities given in Figure 3, Eqs (9) and (10) reduce to

$$
\begin{aligned}
& T_{d}(t)=\frac{F t}{\rho c d}+\frac{F}{k} \frac{A_{d}\left(d-d_{d}\right)\left(1-\exp ^{(-8 a t / A d)}\right)}{8 d_{d} d} \\
& T_{d}(t)-T_{n d}(t)=\frac{F}{k} \frac{A_{d}\left(d-d_{d}\right)\left(1-\exp ^{(-8 a t / A d)}\right)}{8 d_{d} d}
\end{aligned}
$$

Solving Eqs.(12) and (13) results in

$$
T_{n d}(t)=\frac{F t}{\rho c d}
$$

This is similar to Eq. (4), which showed the temperature over the non-defect region and increases linearly with time. After sufficient heating time, depending upon the thermal diffusivity and area of the defect the second term of Eq. (12) will reach the saturation value. Hence, $T_{d}$ increases at the same time as $T_{n d}$, and will result in the saturation of absolute thermal contrast, $\Delta T_{\text {sat }}$, is given by

$$
\Delta T_{s a t}=T_{n d}(t)-T_{d}(t)=\frac{F}{k} \frac{A_{d}\left(d-d_{d}\right)}{8 d_{d} d}
$$

From Eq. 15, absolute thermal contrast can be defined as the arithmetic difference between the temperature in defect and non-defect area. 


\section{Material and Problem Analysis}

In this section, an AISI 316 steel specimen is taken into consideration having a centrally located square flat bottom blind hole with defect $\mathrm{d}_{d}$ and sides $\mathrm{L}_{n d}$ and $\mathrm{L}_{d}$ of non-defect region and defect region respectively. It is clear from Figure 4 , that the side of non-defect region is much larger than the defect region $(\mathrm{A} n d>>\mathrm{A} d)$.

The simulation is performed using the same value of heat flux, temperature and defect size adopted from the experiment. A $5.6 \mathrm{~kW} / \mathrm{m}^{2}$ heat flux is taken to be incident on an AISI 316 steel material with known dimensions of a square defect having a depth of $2 \mathrm{~mm}$ and $3 \mathrm{~mm}$.

The physical properties of the specimen $(\mathrm{k}=16.2 \mathrm{~W} / \mathrm{m}$ $\mathrm{K}, \rho=8000 \mathrm{~kg} / \mathrm{m}^{3}, \mathrm{c}=502 \mathrm{~J} / \mathrm{kg} \mathrm{K}$ ) are used in numerical computation. In order to get uniform emissivity, surface of the sample is coated with carbon to obtain an accurate value for absolute thermal contrast. The surface temperature variations are caused not only because of hidden defects but also by non-uniform heating.
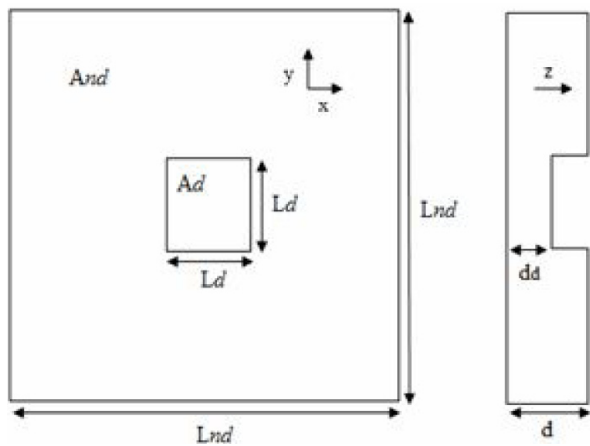

Figure 4. Schematic of AISI 316 steel specimen.

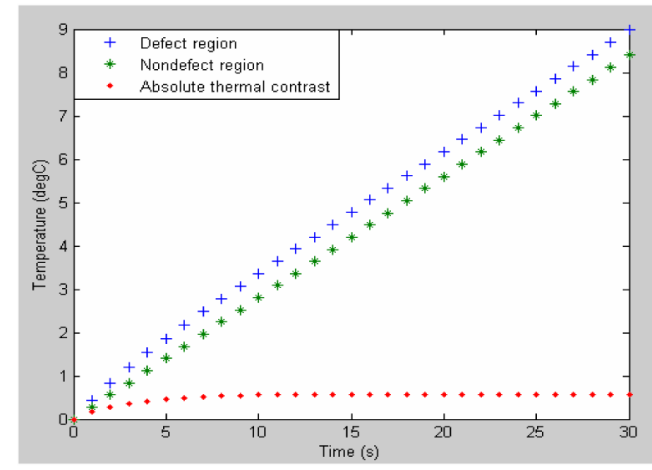

Figure 5. Time Vs. Temperature (3mm).

\section{Results and Discussions}

The simulation has been performed for different depths of defects as a function of time. The analytical expressions derived from the electro thermal model in the previous section have been employed to illustrate the similarity in the temperature and voltage plots shown in the following figures.

From Table 2, it is clear that the values obtained from the simulated plots are nearly equal. The meager difference can be neglected as it arises from the approximation of values obtained using the analytical expressions. The applicability of this solution has been obtained for another depth of 2mm, as in Figure 7-8.

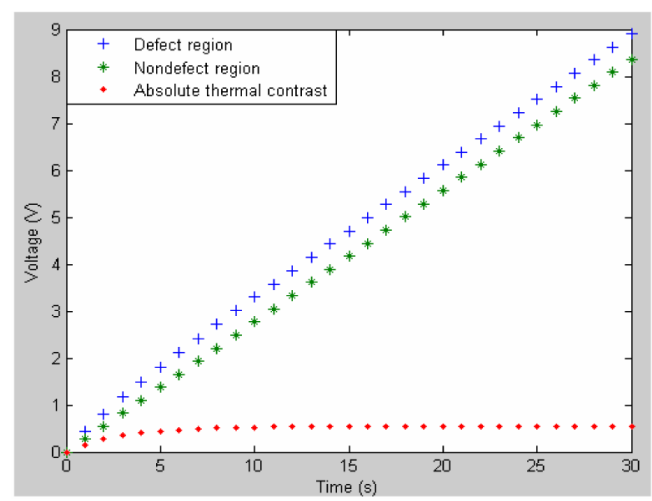

Figure 6. Time Vs Voltage (3mm).

Table 2. Comparison between Temperature and Voltage plots ( $3 \mathrm{~mm}$ defect)

\begin{tabular}{lccc} 
Plots & Defect & Non defect & Thermal contrast \\
\hline Temperature $\left({ }^{\circ} \mathrm{C}\right)$ & 9 & 8.4 & 0.6 \\
Voltage $(\mathrm{V})$ & 8.9 & 8.3 & 0.6 \\
\hline
\end{tabular}

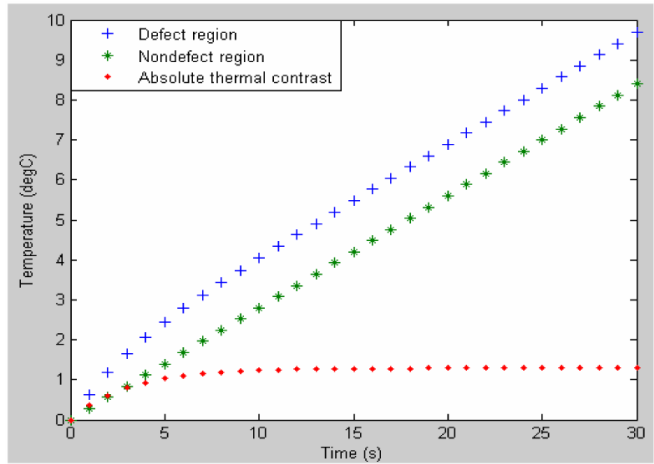

Figure 7. Time Vs Temperature (2mm). 


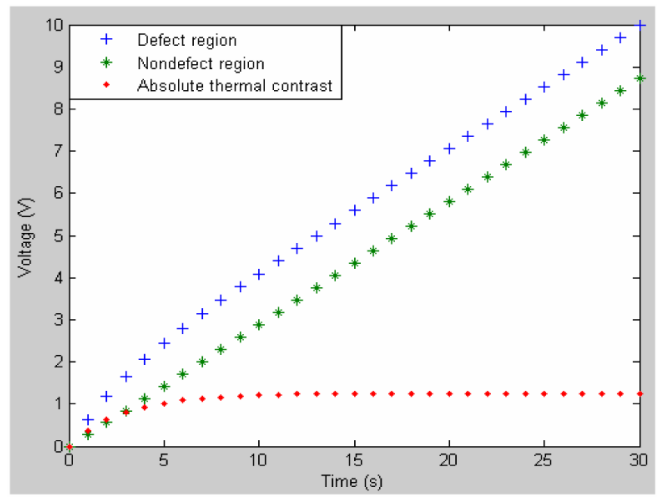

Figure 8. Time Vs Voltage (2mm).

Table 3. Comparison between Temperature and Voltage plots (2mm defect)

\begin{tabular}{llll}
\hline Plots & Defect & Non defect & Thermal contrast \\
\hline Temperature $\left({ }^{\circ} \mathrm{C}\right)$ & 9.8 & 8.5 & 1.3 \\
Voltage $(\mathrm{V})$ & 10 & 8.8 & 1.2 \\
\hline
\end{tabular}

A very interesting behavior of absolute thermal contrast with increasing time can be observed in the plots given. The contrast curve saturates after certain time of heating even though the temperature of the sample increases with heating. This temperature difference (absolute thermal contrast) is also plotted in the figures. The rate of rise of temperature over the etched and non-etched region increases linearly with time at the same rate.

The above results show that the derived analytical expressions for temperature and voltage provide a good estimation. The accuracy of this method mainly depends on the values of the saturated thermal contrast, $\delta \mathrm{T}_{\text {sat }}$. During transient heat conduction, the surface temperature evolution in the defect area will be greater than the reference area, has been observed in the simulated result. However, the study investigates that the temperature distribution over the material varies due to the presence of sub-surface anomalies.

\section{Conclusion}

Transient heat conduction through the material is incorporated in the RC modeling to study the relation between thermodynamic and electrical parameters. In the presented approach, electro-thermal model for a sample material is developed to derive the analytic expression for defect and non-defect temporal regions. As the thickness of the material decreases, the temperature rises in that region than the reference zone and can be observed in the graph. Comparison between the simulated results of temperature and voltage as a function of time shows a good agreement to prove the analogy which validates the present work.

\section{Recommendations}

There is a scope for introducing the effect of other types of heat transfer like convection and radiation. The same electro-thermal approach can be implemented by using other thermographic techniques.

\section{References}

1. Mulaveesala R, Panda S S B et al. (2012). Non-destructive evaluation of concrete structures by non-stationary thermal wave imaging, progress, Electromagnetics Research Letters, vol 32, 39-48.

2. Ghali V S, Mulaveesala R et al. (2011). Frequency modulated thermal wave imaging for non-destructive testing of carbon fiber-reinforced plastic materials, Measurement Science and Technology, vol 22, No. 10, 104018.

3. Gupta R, and Tuli S (2002). Heat pulse optimization by SPICE simulation for transient thermography in silicon Thermosense XXIV, Proceedings of SPIE, vol 4710, 618-625.

4. Maldague X P V (2001). Theory and practice of infrared thermography for nondestructive testing, John Wiley \& Sons, Inc.

5. Gupta R, and Tuli S (2005). Electro-thermal modeling and analysis for estimation of defect parameters by stepped infrared thermography, Elsevier, NDT\&E International vol 38(1), 11-19. 